\title{
Monitoring Menstrual Health in the Sustainable Development Goals
}

\author{
Libbet Loughnan, Thérèse Mahon, Sarah Goddard, Robert Bain, \\ and Marni Sommer
}

\section{Introduction: The Sustainable Development \\ GOALS 2015-2030}

This chapter examines the relationship between menstruation and the Sustainable Development Goals (SDGs), which are the main global development framework for 2015-2030. We examine this relationship by focusing on monitoring progress toward these goals.

The global development community in partnership with national governments and global institutions proposed "a universal call to action to end poverty, protect the planet, and ensure that all people enjoy peace and prosperity" (UNDP 2018, para. 2) as represented by the SDGs, which were adopted by United Nations Member States in 2015. The SDGs "seek to realize the human rights of all and to achieve gender equality and the empowerment of all women and girls" (Sustainable Development Knowledge Platform 2015, 1). They envisage a transformative approach to global development and advancement and have embedded within them the human rights principles of universality, indivisibility, and interdependence (United Nations 2015b, 1). The broad development agenda is codified into 17 SDGs (Fig. 44.1). 


\begin{tabular}{|l|}
\hline Goal 1. No Poverty \\
\hline Goal 2. Zero Hunger \\
\hline Goal 3. Good Health and Well-Being \\
\hline Goal 4. Quality Education \\
\hline Goal 5. Gender Equality \\
\hline Goal 6. Clean Water and Sanitation \\
\hline Goal 7. Affordable and Clean Energy \\
\hline Goal 8. Decent Work and Economic Growth \\
\hline Goal 9. Industry, Innovation and Infrastructure \\
\hline Goal 10. Reduced Inequalities \\
\hline Goal 11. Sustainable Cities and Communities \\
\hline Goal 12. Responsible Consumption and Production \\
\hline Goal 13. Climate Action \\
\hline Goal 14. Life Below Water \\
\hline Goal 15. Life on Land \\
\hline Goal 16. Peace, Justice and Strong Institutions \\
\hline Goal 17. Partnerships for the Goals \\
\hline
\end{tabular}

Fig. 44.1 List of Sustainable Development Goals (Source United Nations Department of Public Information 2019)

\section{Why the SDGs Matter for Menstrual Health}

In evaluating progress on the SDGs, analyzing whether the menstruation needs of populations are being met can yield valuable information. First, the menstrual cycle's regular occurrence between puberty and menopause is an important indicator of good reproductive health. Second, overcoming menstrual-related stigma and ensuring that women and girls can manage their menstruation is key to achieving SDGs that touch on women's and girls' comfort, agency, participation, safety, well-being, and dignity.

The ability of people who are menstruating (for example, adolescent girls, women, and also others who do not identify as women or girls and 
menstruate $\left.^{\mathrm{l}}\right)$ to manage monthly menstruation requires three distinct components: tailored assets (for example, knowledge, confidence, and awareness about how to manage menstruation; an adequate supply of hygienic absorbent materials to collect menstrual blood; water and soap for washing the body), services (for example, information and education), and spaces (for example, safe and convenient sanitation facilities to change and dispose of materials with privacy and dignity as often as necessary for the duration of a menstrual period) (WHO/UNICEF JMP 2015, 45; Patkar 2012). These collective components have been termed "menstrual hygiene management" (MHM) by the global community of experts working to improve access to drinking water, sanitation, and hygiene (the WASH sector). The major domains of life-including home, schools, health centers, and public sanitation facilities - should be supportive of MHM by addressing the need for these assets, services, and spaces. But often they do not. This negatively affects women and girls, and all those who menstruate, throughout their lives. Ongoing stigma around menstruation hinders action on adequately addressing the three components in many contexts, including the perpetuation of many menstrual-related restrictions in certain cultural contexts.

The SDGs are translated into policies and programs at global, national, and local levels; thus, whether the relevant SDGs are supportive of menstruation-related needs or not will be reflected in myriad ways in adolescent girls' and women's lives. As Dr. Jyoti Sanghera, Head of Economic, Social and Cultural Rights at the Office of the High Commissioner for Human Rights, articulates: "Stigma around menstruation and menstrual hygiene is a violation of several human rights," including "the right to human dignity, but also the right to non-discrimination, equality, bodily integrity, health, privacy and the right to freedom from inhumane and degrading treatment from abuse and violence" (George 2013, 5). Menstruation-related tailored assets, services, and spaces are essential to meeting the rights articulated in the SDGs and addressing the persistent stigma women and girls face.

\section{The Scope of This Chapter: Indicators for Monitoring}

The 17 SDGs are represented by 169 targets and 232 indicators. The targets, designed to guide development efforts until 2030, were negotiated by UN member states taking into consideration inputs from a wide range of stakeholders, with progress monitored by one or two globally applicable and measurable indicators per target. The indicators are the main monitoring apparatus of the SDGs, so we focus on them in this chapter. They represent an agreed-upon evidence base by which progress on the targets and goals will be monitored. This monitoring is essential to ensure that real progress is being made at the local, national, and global levels. It also will 
assist governments and development partners with guiding investments and targeted action.

Our analysis provides perspective on the extent to which the indicators adequately capture whether adolescent girls' and women's menstruation-related needs are being met. We also examine the potential of other large-scale menstruation-related monitoring initiatives to help understand whether the SDGs are being achieved and how they might address weaknesses of the existing indicator framework. Through this assessment, we offer a goal-by-goal overview of the national-scale monitoring that substantiates the connections between menstrual health and the SDGs. We do not attempt to assess each of these connections, but rather to identify the strongest links and stimulate discussion so that we may collectively strengthen linkages that will lead to progress on menstrual health and the SDGs.

\section{The Links Between Monitoring Progress Toward the SDGs and Menstruation}

As MHM is not explicitly mentioned in any of the SDGs, targets, or indicators, global development actors need to more explicitly articulate why monitoring on MHM contributes to the assessment of progress on multiple goals. Here we articulate links between menstrual health and SDG indicators under goals that address the following issues: poverty, health, education, gender equality, water and sanitation, and others.

SDG 1, and particularly Target 1.4, recognize the gendered nature of poverty. The phrasing of the corresponding Indicator 1.4.1 is gender-neutral (see Fig. 44.2). However, its definition and monitoring should not be. First, disaggregation by sex is an explicit aim for the monitoring of all

\section{GOAL 1 | END POVERTY IN ALL ITS FORMS EVERYWHERE}

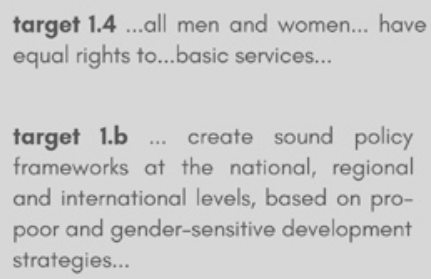

Fig. 44.2 SDG 1 indicators with the highest relevance to menstruation (Source UN Statistics Division [2018]. Design Credit: Sydney Amoakoh 2019) 
SDGs (United Nations 2015b, 37). Second, the metadata explaining the rationale for the indicator recalls that member states commit to "ensuring that these basic services are responsive to the rights and needs of women" (United Nations 2016, 8).

Despite this, the definition of "basic services" does not appear to specify services that directly respond to women's and girl's menstrual hygiene needs. Basic services include: "drinking water, sanitation and hygiene, energy, mobility, waste collection, health care, education and information technologies" (UN Statistics Division 2018). The sanitation ("the use of improved facilities that are not shared with other households") and hygiene ("handwashing facility on premises with soap and water") aspects are linked to SDG 6 definitions of "access to basic services," suggesting that unless these explicitly incorporate services (and assets and spaces) needed to manage menstruation then Goal 1 will fall short of reflecting the basic needs of menstruating girls and women.

Indicator 1.b.1 measures government spending that disproportionately benefits women, which could be interpreted with relevance to menstruation. Increasing government spending on any of the critical components of MHM could be claimed as progress on MHM-for example, dissemination of information about how to manage menstruation. Reducing taxes on sanitary products might also arguably be considered as progress (Fig. 44.3).

The focus of the denominator in Indicator 3.7.1 is the ability to reproduce, using an age bracket typifying the ages women menstruate. The indicator will miss important information about women whose menarche comes earlier, or menopause later. Indicator 3.8.1 brings WASH and health together, with the custodian agency (World Health Organization, or WHO) making an index from 14 indicators of universal health coverage. The

\section{GOAL 3 | ENSURE HEALTHY LIVES \& PROMOTE WELL-BEING FOR ALL AT ALL AGES}

target 3.7...ensure universal access to sexual and reproductive health-care services...including for family planning, information and education, and the integration of reproductive health into national strategies and programmes

target 3.8 Achieve universal health coverage, including...access to quality essential health-care services...for all indicator 3.7.1 Proportion of women of reproductive age (aged 15-49 years) who have their need for planning satisfied with modern methods

indicator 3.8.1 Coverage of essential health services

Fig. 44.3 SDG 3 indicators with the highest relevance to menstruation (Source UN Statistics Division [2018]. Design Credit: Sydney Amoakoh 2019) 


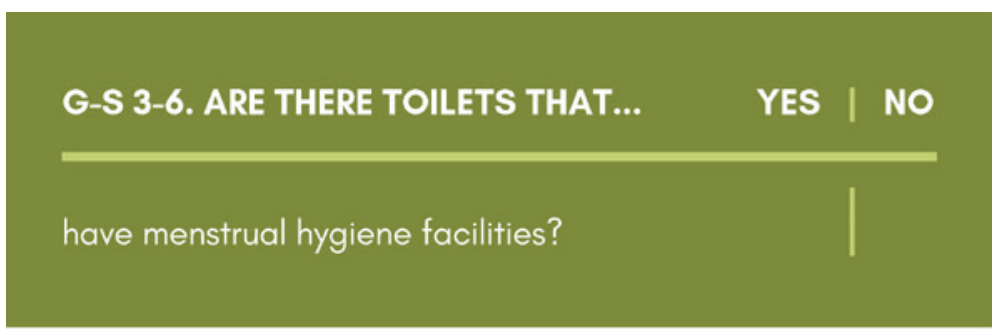

NOTES | A toilet can be considered to have menstrual hygiene facilities if it

- has a bin with a lid on it for disposal of used menstrual hygiene products, and

- water and soap available in a private space for washing

Fig. 44.4 Measurements already operationalized for SDG monitoring in healthcare facilities (Source WHO/UNICEF JMP [2016], 5. Design Credit: Sydney Amoakoh 2019)

metadata notes that the WASH aspect of this broad concept is basic WASH as defined under SDG 6 (UN Statistics Division 2018).

MHM is clearly of utmost importance to women's health, and despite the links above, it is not directly mentioned in SDG 3. It is under other SDGs where the most explicit links between health and menstruation currently sit. Healthcare centers and schools have been identified as places other than home where people spend critical or lengthy periods of time, so they have priority in the monitoring of indicators related to SDG 6, which we discuss further below. In recognition of this, the WHO/UNICEF Joint Monitoring Programme (JMP) is expanding its global databases to include WASH in institutional settings. To be considered to meet the SDG 'basic' sanitation service criteria, healthcare centers are required to have at least one usable improved toilet designated for women and girls, which provides facilities to manage menstrual hygiene (see Figs. 44.4 and 44.5).

SDG 4 focuses on education. United Nations Educational, Scientific and Cultural Organization (UNESCO), the custodian agency for monitoring Indicator 4.a.1 and Indicator 4.3.1, makes it clear that the indicators fall short of representing the relevance menstruation has to the goal. Of the many changes during puberty, UNESCO considers menstruation to have the most pronounced effect on the quality and enjoyment of education (UNESCO $2014,10)$ and that "primary school is [thus] the right place and the right time to reach young people with puberty education" (UNESCO 2014, 10). Adequate assets, services, and spaces for menstrual hygiene are likely to be essential to enable full engagement and concentration (Sommer et al. 2016, 3). However, similar to SDG 1, the metadata sheet notes the "basic" WASH component of Indicator 4.a.l defers to the JMP “(as per the WASH indicator 


\section{GOAL 4 | ENSURE INCLUSIVE \& EQUITABLE QUALITY EDUCATION \& PROMOTE LIFELONG LEARNING OPPORTUNITIES FOR ALL}

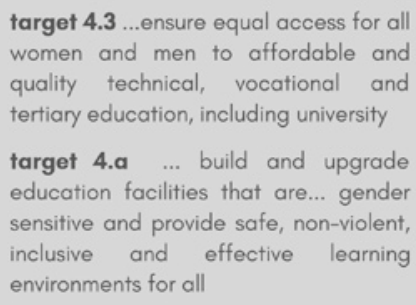

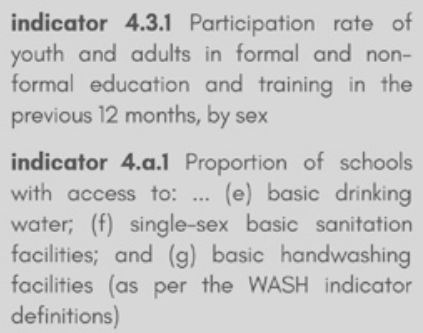

Fig. 44.5 SDG 4 indicators with the highest relevance to menstruation (Source UN Statistics Division [2018]. Design Credit: Sydney Amoakoh 2019)

definitions)"- that is, the proportion of schools with single-sex basic sanitation and handwashing facilities with water and soap. These indicators go some way to understanding whether schools are providing gender-sensitive environments but do not include many relevant aspects of MHM-for example, menstrual hygiene facilities or materials. It should be noted that, in addition to monitoring the "basic" WASH in schools indicators, for contexts where there is a greater capacity for monitoring, the JMP outlines an "advanced service" of sanitation (including menstrual hygiene facilities) and hygiene (including menstrual hygiene materials) in school settings (WHO/UNICEF JMP 2018, 11), accompanied by an expanded set of recommended questions. However, unless the services are considered "basic," they remain outside the cross-cutting SDG monitoring. The metadata sheet for Indicator 4.3.1 does not mention menstruation at all, but the participation rates will be disaggregated by sex, so where inequities are found, stakeholders might do well to check whether inadequate MHM helps explain it (Fig. 44.6).

Indicators and targets for SDG 5 do not explicitly mention menstruation, but that does not mean it is not relevant. As the SDGs have embedded the human rights framework, an understanding of SDG 5 must incorporate the notion of substantive equality, examining both the explicit and direct discriminatory practices by states that treat women and girls differently and also those that may appear gender-neutral yet practically disadvantage women and girls (Winkler and Roaf 2014, 20). Societal taboos and embarrassment around menstruation result in neglect of women's menstrual hygiene needs. This is compounded by gender inequality, which often excludes women and girls from decision-making in development processes (Mahon and Fernandes $2010,100)$. 


\section{GOAL 5 | ACHIEVE GENDER EQUALITY \& EMPOWER ALL WOMEN \& GIRLS}

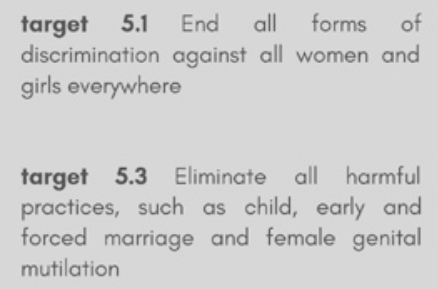

indicator 5.1.1 Whether or not legal frameworks are in place to promote, enforce and monitor equality and nondiscrimination on the basis of sex

indicator 5.3.1 Proportion of women aged 20-24 years who were married or in a union before age 15 and before age 18

Fig. 44.6 SDG 5 indicators with the highest relevance to menstruation (Source UN Statistics Division [2018]. Design Credit: Sydney Amoakoh 2019)

According to custodian agencies of Indicator 5.1.1, national counterparts assess the indicator by scoring on 45 binary yes/no questions. They include, for example, "Does the law mandate nondiscrimination on the basis of gender in employment?" and "Are there laws that explicitly require the production and/or dissemination of gender statistics?" (UN Statistics Division 2018). Being able to answer "yes" to these questions represents progress under this indicator. This bodes well for efforts to increase access to MHM services, assets, and spaces, as the indicator clearly incentivizes generating evidence on gender-based inequality and a legal framework that those perceiving discrimination can seek help from.

Circumstances are not always straightforward, as an example from China illustrates. China's State Council issued the "Special Rules on Labor Protection for Female Employees" in 2012 "to reduce and resolve the special difficulties which female employees may have in the course of their labor due to their physiological characteristics, and to protect their health." The rules define the "scope of prohibited labor for female employees during the menstrual period" (ACFTU 2014, 1). Although phrased about protection, the broad range of prohibited labor may also incentivize an employer to recruit males instead of females (see Levitt and Barnack-Tavlaris in the volume). We hope for healthy debate on what constitutes progress or regression under each of these questions used in monitoring.

Under Indicator 5.3.1, which addresses early marriage, we see a link to menstruation. In some cultures, due to a combination of social or economic reasons and anxieties, menarche can be a trigger for forced or early marriage. Because of the relationship between menstruation and gender inequality, any tracking of improvements in menstruation-related discrimination and provision of services to address adolescent girls' and women's needs could also 


\section{GOAL 6 | ENSURE AVAILABILITY \& SUSTAINABLE MANAGEMENT OF WATER \& SANITATION FOR ALL}

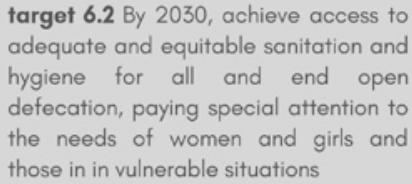
adequate and equitable sanitation and hygiene for all and end open defecation, paying special attention to the needs of women and girls and those in in vulnerable situations

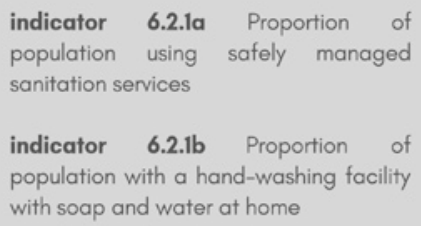

Fig. 44.7 SDG 6 indicators with the highest relevance to menstruation (Source UN Statistics Division [2018]. Design Credit: Sydney Amoakoh 2019)

provide proxy measures for progress toward gender equality. Therefore, the indicators discussed in relation to other SDGs may also relate to SDG 5 (Fig. 44.7).

Target 6.2 has strong relevance to menstrual hygiene: “. . . paying special attention to the needs of women and girls. . ." We argue that this implies addressing the menstrual hygiene needs of women and girls, yet Indicators 6.2.1 a and 6.2.1 b, with a focus on safely managed sanitation services and handwashing facilities at home, fails to address menstrual hygiene directly.

More background may help readers understand more about the SDG 6 wording "paying special attention to the needs of women and girls." During development of the SDG 6 monitoring framework, the JMP convened a technical working group on hygiene that focused on three areas: food hygiene, handwashing with soap, and MHM. The MHM sub-group formulated the following target: "By 2040 all women and adolescent girls are able to manage menstruation hygienically and with dignity." It recommended three MHM indicators: (1) \% of schools and primary health facilities distributing/disseminating accurate, contextually appropriate, pragmatic menstrual management information; (2) \% of teachers and healthcare workers who can answer a set of basic questions on MHM; and (3) \% of public facilities, schools, institutions, transport hubs, and markets that provide gender-separated latrines with water and soap and disposal facilities for menstrual materials (WHO/UNICEF JMP 2016). While the official SDG indicator list did not incorporate these indicators, there has been good progress in monitoring some aspects of these indicators.

The JMP's monitoring already surpasses the limited scope of the official Indicators 6.2.1 a and 6.2.1b. The JMP monitors drinking water, sanitation, 
and hygiene through service "ladders," differentiating more levels or "rungs" of service than called for in the SDG indicators. Most relevant for MHM are the estimates of the proportion of the population practicing open defecation (no sanitation service), the proportion using basic sanitation services, and the proportion with basic hygiene (Loughnan et al. 2016). Not practicing open defecation implies having access to a sanitation facility, which may go some way to offering privacy for changing menstrual materials, part of the critical components of MHM. The use of basic or safely managed sanitation services (by definition not shared with other households) may increase the likelihood women and girls have private facilities for MHM.

Indicator $6.2 .1 \mathrm{~b}$ focuses on the presence of basic hygiene-here, handwashing facilities on the premises with soap and water available. The indicator can provide information on MHM, but only partially so. It suggests an adolescent girl or woman would have some access to water and soap, two of the critical components of MHM. However, having a place for general handwashing is not the same as having sufficient water, soap, and space for washing and bathing themselves and their menstrual materials.

As mentioned under the section on SDG 3, the JMP's recommendations for SDG monitoring in health care settings explicitly includes in "basic" sanitation tracking that toilets must be ". . . separated for women, [and] provide menstrual hygiene facilities. .." (WHO/UNICEF JMP 2017b, 46). In school settings, the JMP considers menstrual hygiene facilities, but only in monitoring at an "advanced" level of access. These healthcare centers and school-specific applications are the only, and limited, instances in the SDG monitoring framework where menstruation is directly addressed.

This still falls short of monitoring the intended scope of the SDG target. Monitoring whether women and girls have the necessary information, confidence, and awareness about how to manage their menstruation-and an adequate supply of hygienic absorptive materials-would also need to be monitored to pay "special attention to the needs of women and girls" in ensuring "adequate and equitable sanitation and hygiene for all."

\section{Other SDG Targets}

At-scale monitoring of menstrual health may hold benefits for understanding changes in a range of indicators under other SDGs. Due to space constraints, we give just a few brief additional examples. Goal 10, the "inequality" goal, brings in a range of indicators examining whether opportunities and participation is leveling out between traditionally disadvantaged and advantaged populations. Some of the monitoring explained above would be relevant under the broad theme of SDG 10. The SDG 10 inequality indicator we examined was Indicator 10.3.1: "Proportion of population reporting having personally felt discriminated against or harassed in the previous 12 months on the basis of a ground of discrimination prohibited under international human rights law" (UN Statistics Division 2018). Discrimination based 
on an individual's sex is prohibited under international human rights law. Some nationally representative surveys like Nepal Multiple Indicator Cluster Surveys (MICS) 2014 survey have included questions to understand discriminatory practices against menstruating women (Central Bureau of Statistics $2015,151)$. Such findings might be recognized under monitoring Indicator 10.3.1 (methodology for this indicator is under development).

Indicator 1.5.3, 11.B.1, and 13.1.2 are all "Number of countries that adopt and implement national disaster risk reduction strategies in line with the Sendai Framework for Disaster Risk Reduction 2015-2030" (UN Statistics Division 2018). The Sendai Framework was developed to reduce risks and losses stemming from disasters and to "Build Back Better." The framework explicitly recognizes that women are disproportionately affected in disaster settings and requires "all-of-society engagement and partnership" that pays "special attention to people disproportionately affected by disasters" (United Nations 2015a, 13). Omitting MHM needs in an emergency would be a failure to meet the specifications of the Sendai Framework.

As our findings across this chapter highlight, there are multiple linkages between menstruation and gender inequalities, and these occur across different domains, including poverty, health, education, and WASH. As menstruation is a biological characteristic of women and adolescent girls, SDG 5 may be the fulcrum around which other levers for change relating to social and biological experiences of menstruation coalesce.

\section{Main Sources of Primary Data on Menstrual Health}

The earlier sections of this chapter moved systematically through the global SDG framework to examine links with menstruation and noted the limited extent to which the SDG indicator framework addresses the menstrual hygiene needs of women and girls. This section describes some of the major data collection pioneers implementing at-scale monitoring of menstruation-related indicators, which can aid understanding of SDG progress, fill gaps in the indicator framework under target 6 , and support monitoring of SDGs 1 and 4 . However, it will be some time before a sufficient number of surveys are available to allow systematic reporting for a reasonable number of countries over time.

\section{Data About the General MHM Circumstances of Women and Girls}

UNICEF-supported MICS have been conducted in over 100 countries and cover a wide range of topics focusing on children's and women's health and well-being. MICS already includes a set of questions that can be used to collect data for SDG Indicators 6.2.1a and 6.2.1b. They have also long asked questions of mothers on whether their menstrual period has returned since giving birth and on the existence and date of their last period (WHO/ UNICEF JMP 2017a, 16). In collaboration with the WHO/UNICEF JMP 
and in consultation with MHM experts, MICS have now included new questions on menstrual hygiene in surveys conducted since 2017. As of February 2019 , there are over 60 MICS surveys at various stages of planning, fieldwork, or analysis that will include these new questions (UNICEF 2019). The questions, based on a pilot in Belize (Khan et al. 2017), focus on the ability to wash and change in privacy at home and access to appropriate reusable or single-use menstrual hygiene materials - two of the essential elements of MHM - as well as participation in social activities, school, or work during a women's menstrual period. The questions pertain to multiple SDGs and hence collect new evidence for monitoring these elements. Furthermore, the datasets contain a wealth of information that can readily be analyzed in conjunction with the menstrual well-being variables.

The Performance Monitoring and Accountability 2020 (PMA2020) surveys led by Johns Hopkins University have been conducted in 11 countries and cover two main topics: family planning and WASH (PMA2020 2018, 9). These surveys have a greater emphasis on MHM and have included a broad evolving range of questions on menstrual hygiene including the main environments for MHM, safety, cleanliness and privacy of MHM facility, disposal of materials, and washing and drying of reusable materials. The latest questionnaires also ask women to recall their age of menarche (Sommer 2013, 401). If PMA2020 consistently collects good-quality data, this may represent a great source for monitoring those essential aspects.

While these questions are standard in the MICS and PMA2020 survey networks, other nationally representative surveys have the option to include them. Household surveys may further offer an opportunity to examine the costs of accessing materials for managing menstruation. A recent review by the International Household Survey Network (IHSN) of income and expenditure surveys for 100 countries found almost all surveys include some information on personal care products (for instance, soap or sanitary pads) (WHO/UNICEF JMP 2017b; IHSN 2019). A lack of consistency across the existing datasets makes them unamenable to the production of comparable international estimates.

\section{Data About the MHM Provisions at Schools and Healthcare Centers}

As noted earlier, WASH in schools and healthcare centers have been identified as priorities for monitoring of SDGs 4 and 6. In August 2018, the JMP released a baseline assessment of WASH in Schools, finding that "few countries had data on the proportion of schools providing menstrual hygiene management education, sanitary towels and facilities for the disposal of used materials" (WHO/UNICEF JMP 2018, 8, 50). Nevertheless, a number of countries have already adopted the JMP-recommended questions for schools, either integrating these questions in Education Management Information Systems (EMIS) (for example, Papua New Guinea) or conducting dedicated 
surveys (for example, in Liberia, and Nigeria's WASH Norm 2018), and more are expected to in the near future.

The main international surveys of health centers include WHO's Service Availability and Readiness Assessment (SARA), DHS's Service Provision Assessment (SPA), and the World Bank's Service Delivery Indicators (SDI) (UNICEF and WHO 2015). They include questions about the availability of water and sanitation facilities in health centers and would be the logical existing mechanisms for collecting this data. Now that consensus and clear collection and analysis methods have been defined, it is expected that an increasing number of these surveys, along with national Health Management Information Systems, will begin to use the question noted in Fig. 44.4 and generate critical information on MHM in the coming years.

\section{New Forms of Data to Aid Understanding of SDG Progress}

In the run-up to Menstrual Hygiene Day 2017, UNICEF polled users of U-report about menstrual hygiene across 19 countries (Wijesekera 2017, para. 8). Such data are not representative, as a phone or internet access was needed for participation, but they may provide a useful "snapshot" of the situation that is relevant across the SDGs, particularly to SDGs 4,5 , and 6.

\section{LOOKING FORWARD}

Although the current global SDG monitoring framework does not comprehensively address the full range of essential MHM components, this chapter makes clear how related monitoring efforts and a nuanced interpretation of the existing targets and indicators can support progress in addressing women's and girls' unmet menstrual hygiene needs. Existing and emerging menstruation-related monitoring could be more effectively used to understand changes measured by a range of SDG indicators and to address their limitations - and a large number of countries are preparing to collect data on menstrual health in the coming years. There is an urgent need for cross-sectorial discussions and action based on the evidence consolidated here to conduct further inquiry into the intersection between the topics of menstruation and the SDGs with an explicit focus on what is monitorable. Furthermore, there is a need to develop monitoring approaches for the essential elements of menstrual hygiene that have yet to be included in monitoring mechanisms (the availability of information about how to manage menstruation, as well as confidence and awareness). With concerted efforts to roll out standardized data collection on all elements of menstrual hygiene, in a few years it may be possible to compare progress between and within countries with a view to tracking progress on menstrual hygiene needs.

We hope this chapter helps breathe new life into the discussion on menstrual hygiene and sustainable development targets. As the SDG indicators 
are adopted more widely and more data are amassed and interpreted, the generation of sex-disaggregated data under all indicators may itself provide additional unanticipated utility for understanding whether menstruation management needs are being met in the specific setting. The many connections between MHM and the range of SDGs will likely become even more evident.

\section{Note}

1. The scope of this chapter is limited, focusing on monitoring menstrual hygiene for women and adolescent girls. To the extent that the content is useful to others who menstruate, we would encourage a more inclusive interpretation of the text. The full spectrum of people menstruating needs to be addressed with dedicated research, policy, and programming beyond the scope of this chapter.

\section{REFERENCES}

ACFTU. 2014. "Special Rules on the Labor Protection of Female Employees (2012)." All-China Federation of Trade Unions (Blog), August 26. http:// en.acftu.org/28616/201408/26/140826131330762.shtml.

Central Bureau of Statistics. 2015. "Nepal Multiple Indicator Cluster Survey 2014." Kathmandu, Nepal: Central Bureau of Statistics and UNICEF Nepal. https:// mics-surveys-prod.s3.amazonaws.com/MICS5/South\%20Asia/Nepal/2014/ Final/Nepal\%202014\%20MICS_English.pdf.

George, Rose. 2013. "Celebrating Womanhood: How Better Menstrual Hygiene Management Is the Path to Better Health, Dignity and Business." Geneva: Water Supply and Sanitation Collaborative Council. http://wsscc.org/wp-content/ uploads/2016/05/Celebrating-Womanhood-How-better-menstrual-hygienemanagement-is-the-path-to-better-health-dignity-and-business-\%E2\%80\%93WSSCC-2013.pdf.

International Household Survey Network (IHSN). 2019. "Measuring Non-Food Expenditures." IHSN. http://www.ihsn.org/projects/non-food-assessment.

Khan, Shane M., Robert E. S. Bain, Karsten Lunze, Turgay Unalan, Bo Beshanski-Pedersen, Tom Slaymaker, Richard Johnston, and Attila Hancioglu. 2017. "Optimizing Household Survey Methods to Monitor the Sustainable Development Goals Targets 6.1 and 6.2 on Drinking Water, Sanitation and Hygiene: A Mixed-Methods Field-Test in Belize." Edited by Asim Zia. PLoS One 12 (12): e0189089. https://doi.org/10.1371/journal.pone.0189089.

Loughnan, Libbet C., Rob Bain, Rosemary Rop, Marni Sommer, and Tom Slaymaker. 2016. "What Can Existing Data on Water and Sanitation Tell Us about Menstrual Hygiene Management?” Waterlines 35 (3): 228-44. https://doi. org/10.3362/1756-3488.2016.019.

Mahon, Thérèse, and Maria Fernandes. 2010. "Menstrual Hygiene in South Asia: A Neglected Issue for WASH (Water, Sanitation and Hygiene) Programmes." Gender \& Development 18 (1): 99-113. https://doi.org/10.1080/13552071003600083.

Patkar, Archana. 2012. "Preparatory Input on MHM for JMP Equity and Non-Discrimination Working Group by Archana Patkar." Water Supply and Sanitation Collaborative Council (WSSCC). 
PMA2020. 2018. "Performance Monitoring and Accountability 2020: Measuring Performance, Informing Policy, Empowering Communities." Report Summary. Baltimore, MD: Johns Hopkins University and Bill and Melinda Gates Foundation. https://www.pma2020.org/sites/default/files/PMA2020_Brochure_100818_ SPREADS.pdf.

Sommer, Marni. 2013. "Menarche: A Missing Indicator in Population Health from Low-Income Countries.” Public Health Reports 128 (5): 399-401. https://doi. org/10.1177/003335491312800511.

Sommer, Marni, Bethany A. Caruso, Murat Sahin, Teresa Calderon, Sue Cavill, Therese Mahon, and Penelope A. Phillips-Howard. 2016. "A Time for Global Action: Addressing Girls' Menstrual Hygiene Management Needs in Schools." PLOS Medicine 13 (2): el001962. https://doi.org/10.1371/journal. pmed.1001962.

Sustainable Development Knowledge Platform. 2015. "Transforming Our World: The 2030 Agenda for Sustainable Development.” United Nations. https://sustainabledevelopment.un.org/post2015/transformingourworld.

UN Statistics Division. 2018. "SDG Indicators Metadata Repository." SDG Indicators, October 2018. https://unstats.un.org/sdgs/metadata/.

UNDP. 2018. "What Are the Sustainable Development Goals?" Sustainable Development Goals, 2018. https://www.undp.org/content/undp/en/home/ sustainable-development-goals.html.

UNESCO. 2014. Puberty Education \& Menstrual Hygiene Management. Good Policy and Practice in Health 9. Paris: United Nations Educational, Scientific and Cultural Organization. http://unesdoc.unesco.org/images/0022/002267/226792e.pdf.

UNICEF. 2019. "MICS 6 Surveys.” http://mics.unicef.org/surveys.

UNICEF and WHO. 2015. "Water, Sanitation and Hygiene in Health Care Facilities: Status in Low- and Middle-Income Countries and Way Forward.” UNICEF and WHO.

United Nations. 2015a. "Sendai Framework for Disaster Risk Reduction 20152030." New York: United Nations. http://www.unisdr.org/files/43291_sendaiframeworkfordrren.pdf.

—. 2015b. "Transforming Our World: The 2030 Agenda for Sustainable Development." New York: United Nations. https://sustainabledevelopment. un.org/content/documents/2 1252030\%20Agenda\%20for\%20Sustainable $\% 20$ Development\%20web.pdf.

- 2016. Resolution Adopted by the General Assembly on 23 December 2016. http://www.un.org/en/development/desa/population/migration/generalassembly/docs/globalcompact/A_RES_71_256.pdf.

United Nations Department of Public Information. 2019. Sustainable Development Goals. https://sustainabledevelopment.un.org/sdgs.

WHO/UNICEF JMP. 2015. Progress on Sanitation and Drinking Water: 2015 Update and MDG Assessment. Geneva: World Health Organization and United Nations Children's Fund.

- 2016. "Monitoring WASH in Health Care Facilities: Final Core Indicators and Questions." World Health Organization and UNICEF. https://washdata. org/report/jmp-2016-core-questions-and-indicators-monitoring-winhcf.

. 2017a. "MICS6 Questionnaire for Individual Women." UNICEF Multiple Indicator Cluster Surveys. http://mics.unicef.org/files?job=WIsiZiIsIjIwMTcvMTEvMzAvMTkvMTIvNDIvNDg5L01JQ1 M2X1F1ZX- 
N0aW9ubmFpcmVfZm9yX0luZG12aWRlYWxfV29tZW5fMjAxNzExMjkuZG9jeCJdXQ\&sha=6328aec5f4566776.

.2017b. Progress on Drinking Water, Sanitation and Hygiene: 2017 Update and SDG Baselines. Geneva: World Health Organization and United Nations Children's Fund.

- 2018. "Drinking Water, Sanitation and Hygiene in Schools." Global Baseline Report 2018. New York: UNICEF and WHO. https://data.unicef.org/wp-content/ uploads/2018/08/JMP-WASH-in-Schools-WEB.pdf.

Wijesekera, Sanjay. 2017. "\#MenstruationMatters . . to All of Us.” HuffPost (Blog), May 27, 2017. https://www.huffingtonpost.com/entry/menstruationmatters-toall-of-us_us_59298550e4b07d848fdc0415.

Winkler, Inga T., and Virginia Roaf. 2014. "Taking the Bloody Linen Out of the Closet: Menstrual Hygiene as a Priority for Achieving Gender Equality." Cardozo Journal of Law and Gender 21 (1): 1-37.

Open Access This chapter is licensed under the terms of the Creative Commons Attribution 4.0 International License (http://creativecommons.org/licenses/ by $/ 4.0 /$ ), which permits use, sharing, adaptation, distribution and reproduction in any medium or format, as long as you give appropriate credit to the original author(s) and the source, provide a link to the Creative Commons license and indicate if changes were made.

The images or other third party material in this chapter are included in the chapter's Creative Commons license, unless indicated otherwise in a credit line to the material. If material is not included in the chapter's Creative Commons license and your intended use is not permitted by statutory regulation or exceeds the permitted use, you will need to obtain permission directly from the copyright holder.

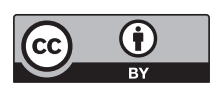

